\title{
Blind Void Filling in LR-EPONs: How Efficient It Can Be?
}

\author{
Amr Elrasad and Basem Shihada \\ Computer, Electrical and Mathematical Sciences and Engineering Division, \\ King Abdullah University of Science and Technology (KAUST), \\ Thuwal, Saudi Arabia \\ Email: \{amr.rasad, basem.shihada\}@kaust.edu.sa
}

\begin{abstract}
This work proposes a novel blind void (idle periods) filling in Long-Reach Ethernet Passive Optical Networks (LREPONs) namely Size Controlled Batch Void Filling (SCBVF). We emphasize on reducing grant delays and hence reducing the average packet delay. SCBVF delay reduction is achieved by early flushing data during the idle time periods (voids) between allocated grants. The proposed approach can be integrated with almost all of the previously reported dynamic bandwidth allocation schemes. SCBVF is less sensitive to differential distance between ONUs and can work well in case of small differential distances compared to previously reported void filling schemes. We support our work by extensive simulation study considering bursty traffic with long range dependency. Numerical results show a delay reduction up to $35 \%$ compared to non-void filling scheme outperforming its main competitors that can achieve up to $7 \%$ delay reduction.
\end{abstract}

\section{INTRODUCTION}

EPON is one of the promising solutions to satisfy the increasing bandwidth demand in access network. It can support a transmission capacity up to $10 \mathrm{Gbps}$ in both upstream and downstream directions. Upstream bandwidth assignment in EPONs is implemented using polling strategy to eliminate collisions. The optical-line-terminal (OLT) unit polls the opticalnetwork-units (ONUs) to transmit according to a certain order. Polling can either be online (interleaved) or offline. In online polling, the grant assignment is carried on immediately after the OLT receives bandwidth request from ONU. On the other hand, the OLT waits until it receives all bandwidth requests before granting the assignment in offline polling. The notations used hereafter are shown in Table I.

Dynamic bandwidth allocation (DBA) is the most vital component in EPON as the network performance heavily relies on it. DBA consists of two main functions namely grant sizing and grant scheduling. Grant sizing determines the amount of bandwidth grant assigned to each ONU during cycle. This decision is based on the bandwidth requests sent from ONU via a report message during the previous grant. Most of the proposed DBA schemes use a limited grant sizing policy in which the grant assigned can not exceed a certain threshold $G_{i}^{\max }$ [1]. Grant scheduling determines both start and end time of bandwidth grant. The most common scheduling approach is to use the horizon (first free) time or non-void filling (NVF) as shown in Fig.1. Assuming cyclic polling, grant start time is given by,

$$
\begin{aligned}
& t_{s}(i, n)= \\
& \begin{cases}\max \left(t_{h}(N, n-1), t_{s}^{\min }(i, n)\right) & i=1, \\
\max \left(t_{h}(i-1, n), t_{s}^{\text {min }}(i, n)\right) & 1<i \leq N,\end{cases}
\end{aligned}
$$

The packet delay components $W_{\text {poll }}, W_{\text {grant }}$, and $W_{\text {queue }}$

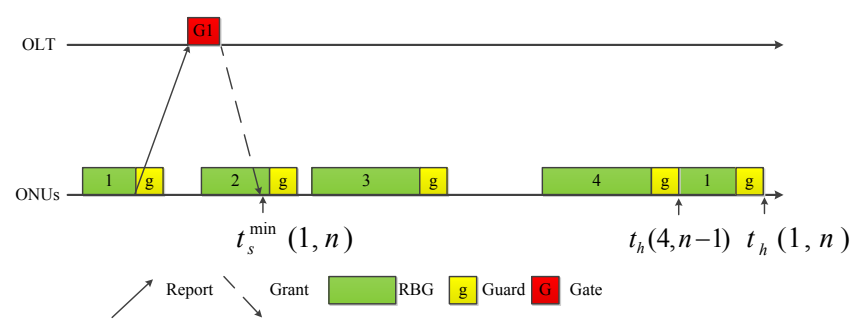

Fig. 1: Bandwidth allocation without void filling (NVF).

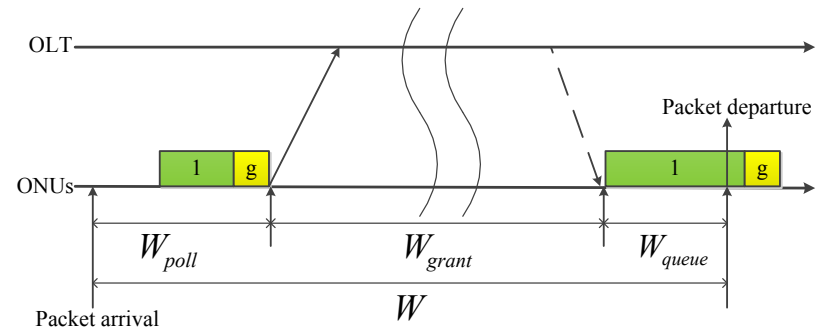

Fig. 2: Delay composition in EPON.

are shown in Fig.2. $W_{\text {poll }}$ is the delay between packet arrival and bandwidth request transmission. On average, $W_{\text {poll }}$ equals half the duration of the cycle time. $W_{\text {grant }}$ is the delay between grant request and grant assignment for certain packet. $W_{\text {grant }}$ spans over multiple cycles but it can not be less than $R T T_{i}+t_{c}$. Hence the minimum grant start time is

$$
t_{s}^{\text {min }}(i, n)=t_{e}(i, n-1)+R T T_{i}+t_{c} .
$$

$W_{\text {queue }}$ is the delay between grant start and packet transmission and it depends on both the buffer size seen by the packet, the grant size, and the packet transmission discipline. 
In general we state that

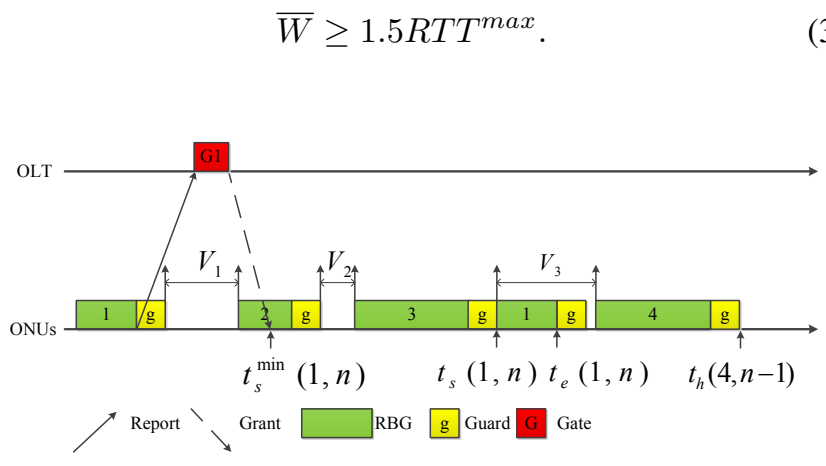

Fig. 3: Request based void filling (RBVF).

In long-reach Ethernet Passive Optical Networks (LREPONs), the distance between OLT and ONUs spans over a longer distance (up to $100 \mathrm{Km}$ ) and hence longer $R T T_{i}$. This is reflected on both $W_{\text {grant }}$ and void time periods between grants [2]. In such a case, $R T T_{i}$ will be the dominant factor in controlling the packet delay at low and medium load [3] such that

$$
\bar{W} \approx 1.5 R T T^{\max }
$$

The negative effect of such voids motivates many researchers to either reduce their size by proposing new DBA schemes [4], [2] or trying to fill these voids with request based bandwidth grants [5], [6]. The void filling techniques presented so far are request based void filling (RBVF). In other words, they rely on fitting a bandwidth grant into one or more of the available voids. In this paper, we propose a novel void filling scheme namely Size Controlled Batch Void Filling (SCBVF) scheme to allocate bandwidth grants to ONUs during voids. Upon void detection, the OLT fills the detected void with bandwidth grants baseless of previous ONUs bandwidth requests. This would help transmitting more packets during unused bandwidth voids and reducing $W_{\text {grant }}$, as well as overall average delay. In order to distinguish between grant types, we will refer to grants based on bandwidth requests as request based grants (RBG), while the grants assigned during voids baseless of bandwidth request will be referred to as void based grants (VBG).

The rest of the paper is organized as follows. Section II introduces the related void filling schemes. Size Controlled Batch Void Filling is presented in section III. Section IV is devoted for comparison discussion between RBVF, RBPVF and SCBVF approaches. Performance evaluation is presented in section V. Finally, conclusions are drawn in section VI.

\section{RELATED WORK}

In [5], a request based void filling was proposed to fit grant requests from relatively nearer ONUs into available voids as shown in Fig.3. Upon receiving bandwidth request and employing grant sizing policy to determine $G(i, n)$, the OLT begins to search for an eligible void that fits the bandwidth grant. A void $V_{k}$ is considered suitable if it matches two

\section{TABLE I: Definition of Variables}

\begin{tabular}{l|l}
\hline$N$ & Number of ONUs, indexed $i=1,2, \ldots, N$ \\
$C$ & Transmission capacity in bps \\
$T_{\text {cycle }}^{\text {max }}$ & Maximum cycle time \\
$G_{i}^{\text {max }}$ & Maximum allocated grant per cycle \\
$R(i, n)$ & ONU $i$ bandwidth request for cycle $n$ \\
$G(i, n)$ & ONU $i$ bandwidth grant for cycle $n$ \\
$w_{i}$ & ONU $i$ weight according to service level agreement (SLA) \\
$t_{g}$ & Guard time between consecutive grants \\
$t_{c}$ & time needed to transmit Gate or report message \\
$D_{i}$ & Distance between OLT and ONU $i$ \\
$R T T_{i}$ & Round-trip-time delay between OLT and ONU $i$ \\
$R T T$ & Average round-trip-time delay \\
$R T T$ max & Maximum round-trip-time delay \\
$t_{s}(i, n)$ & ONU $i$ RBG start time in cycle $n$ \\
$t_{e}(i, n)$ & ONU $i$ RBG end time in cycle $n$ \\
$t_{s}^{\text {min }}(i, n)$ & ONU $i$ RBG minimum start time in cycle $n$ \\
$t_{h}(i, n)$ & The horizon time after ONU $i$ grant allocation in cycle $n$ \\
$V_{i}$ & Void succeeding ONU $i$ RBG \\
$V_{s}(i, n)$ & Start time of void succeeding ONU $i$ RBG \\
$V_{e}(i, n)$ & End time of void succeeding ONU $i$ RBG \\
$W$ & Packet delay \\
\hline$W$ & Average packet delay \\
$W_{\text {poll }}$ & Polling delay \\
$W_{\text {grant }}$ & Grant delay \\
$W_{q u e u e}$ & Queuing delay \\
$V_{i}^{\text {max }}$ & The maximum grant size allocated during VBG for SCBVF \\
\hline
\end{tabular}

conditions described as follows,

$$
V_{e}(k, n)-V_{s}(k, n) \geq G(i, n)
$$

and

$$
V_{e}(k, n)-G(i, n) \geq t_{s}^{\min }(i, n) .
$$

The grant start and end times if $V_{k}$ is chosen are given by

$$
t_{s}(i, n)=\max \left(t_{s}^{\min }(i, n), V_{s}(k, n)\right)
$$

and

$$
t_{e}(i, n)=t_{s}(i, n)+G(i, n) .
$$

The selected void is the one that has minimum $t_{s}(i, n)$ among the set of eligible voids. The computation complexity of RBVF is $O(N)$. If binary search tree is used, the computation complexity is reduced to $O(\log N)$ [5]. The results in [5] show that RBVF reduces the average delay compared to NVF. However, it does not reduce the average delay below the $1.5 \overline{R T T}$ bound, since in the best cases each ONU $i$ can not transmit less than every $R T T_{i}$. RBVF performance improvement decreases with ONUs with small distance variations. If all ONUs have the same $D_{i}$, RBVF can not reduce the average delay compared to NVF [5].

In [6], authors proposed a request based partial void filling (RBPVF) scheme to further improve RBVF. Their algorithm is a mix between RBVF and multi-thread polling (MTP) [4]. Upon receiving bandwidth request, the OLT invokes RBVF to fit the grant within one of the available voids. If the OLT can not find suitable void, it invokes RBPVF that can divide $G(i, n)$ into at most $P$ chunks and switch the corresponding ONU to multi-thread mode. During multi-thread mode, the OLT can not invoke RBPVF and it can use RBVF only. 
The OLT starts eliminating the threads that are requesting zero bandwidth until there is only one thread left. The OLT switches this ONU back to single-thread mode again. This method enables long grants that do not fit in a single void to be divided into two or more grants. Their results shows a delay improvement compared to EFT-RBVF [5], but the minimum bound can be reached is $1.5 \overline{R T T}$ for the same reasons listed above.

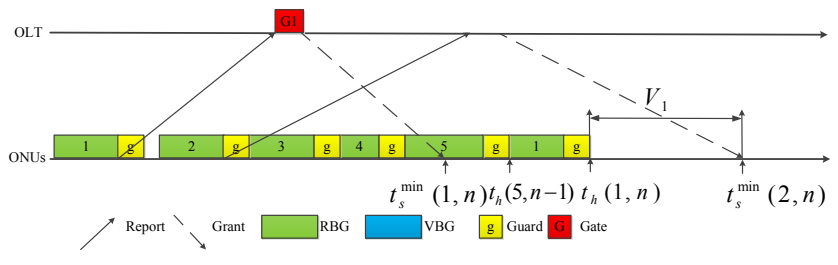

Fig. 4: Void detection.

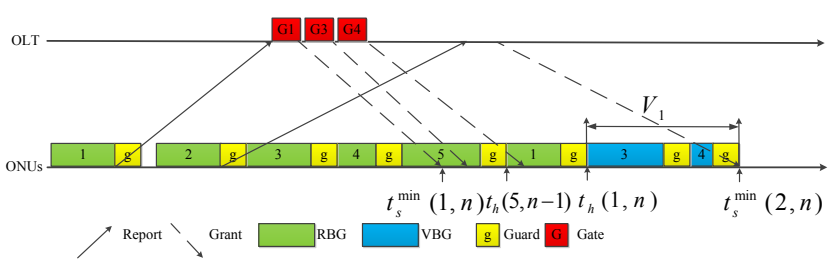

Fig. 5: Size controlled batch void filling (SCBVF).

\section{Size Controlled Batch Void FiLling}

Size Controlled Batch Void Filling (SCBVF) [7] relies on void detection after an RBG is assigned by the OLT as shown in Fig.4. Upon receiving grant request from ONU $i$, the OLT schedules the RBG grant start time as in (1) and updates the horizon time, $t_{h}(i, n)$. In order to detect if there is a void succeeding ONU $i$, the OLT compares $t_{h}(i, n)$ with $t_{s}^{\min }(i+$ $1, n)$. If

$$
t_{s}^{\min }(i+1, n)-t_{h}(i, n)>t_{c}+t_{g},
$$

then the OLT detects a void, $V_{i}$, with start and end times as,

$$
V_{s}(i, n)=t_{h}(i, n)
$$

and

$$
V_{e}(i, n)=t_{s}^{\min }(i+1, n) .
$$

If $i=N$, then the above equations are modified to,

$$
\begin{gathered}
t_{s}^{\min }(1, n+1)-t_{h}(N, n)>t_{c}+t_{g}, \\
V_{s}(N, n)=t_{h}(N, n)
\end{gathered}
$$

and

$$
V_{e}(N, n)=t_{s}^{\min }(1, n+1) .
$$

Upon void detection, The OLT invokes SCBVF immediately to fill the detected voids with void based grant(s) VBG(s). Since ONUs are polled in cyclic order, the void detection computation complexity is of $O(1)$. The size of VBGs only depends on the detected void duration. It is worth noting that during VBG, the ONU will not send bandwidth requests. In fact, bandwidth requests are only sent during RBG. SCBVF assigns VBGs based on maximum predetermined size. In SCBVF, each VBG does not exceed a certain threshold $V_{i}^{\max }$ which is set based on each ONU relative weight $w_{i}$ such that,

$$
\frac{V_{i}^{\max }}{V_{j}^{\max }}=\frac{w_{i}}{w_{j}},
$$

where $i$ and $j \in\{0,1,2, \ldots, N-1\}$. SCBVF continues to allocate VBGs with the maximum void duration until a polled ONU can not be granted its maximum void duration. In order to maximize the benefit of void filling, this ONU will be granted the remaining grant rather that leaving it unscheduled. The operation of SCBVF is shown in Fig.5. In this figure, we observe that ONU 3 has been allocated it's maximum VBG size while ONU 4 was granted the remaining part.

\section{DISCUSSION}

In this section, we discuss the relevant compatibility and overhead factors that is associated with the void filling schemes.

\section{A. Compatibility With Dynamic Bandwidth Allocation}

SCBVF can be integrated with almost all proposed DBA schemes for LR-EPONs. For offline (interleaved-polling-withstop) DBA schemes, SCBVF can be used with offline multithread polling (MTP-offline) [4], offline single thread (STPoffline) [4], and Double Phase (DP) polling [8]. It also can be used with online (interleaved) polling such as online multi-thread polling (MTP-online) [2], online single thread polling with online excess allocation (STP-online-excess) [2], or Delayed Excess Scheduling (DES) [9]. The results in [2] shows that STP-online-excess outperforms MTP-offline and MTP-online at $T_{\text {cycle }}^{\max }$ up to $4 \mathrm{~ms}$. Moreover, STP-online-excess is not as complex as multi-thread polling in terms of reporting process and thread tuning. Although MTP-online has less void size since there is $\Theta N$ RBGs per cycle for $\Theta$ threads. This implies that the cycle contains almost the same idle time amount but divided into more slots. For offline schemes, where there is a single large void at the end of cycle, SCBVF seems to be a reasonable option in that case as it will utilize the large void with the maximum possible VBGs.

\section{B. Control Overhead Proliferation}

Control message proliferation is considered as a side effect of void filling techniques in both upstream and downstream directions. RBVF slightly increases both report and gate messages load. On the other hand, RBPVF increases the report message load more than $\mathrm{RBVF}$ as each partial void grant should end with a report message. SCBVF proposed approaches do not increase the report message load as VBGs do not conclude with a report message. In downstream direction, the amount of increase depends on $V_{i}^{\max }$. It is also affected by the offered load which controls the average void duration. 


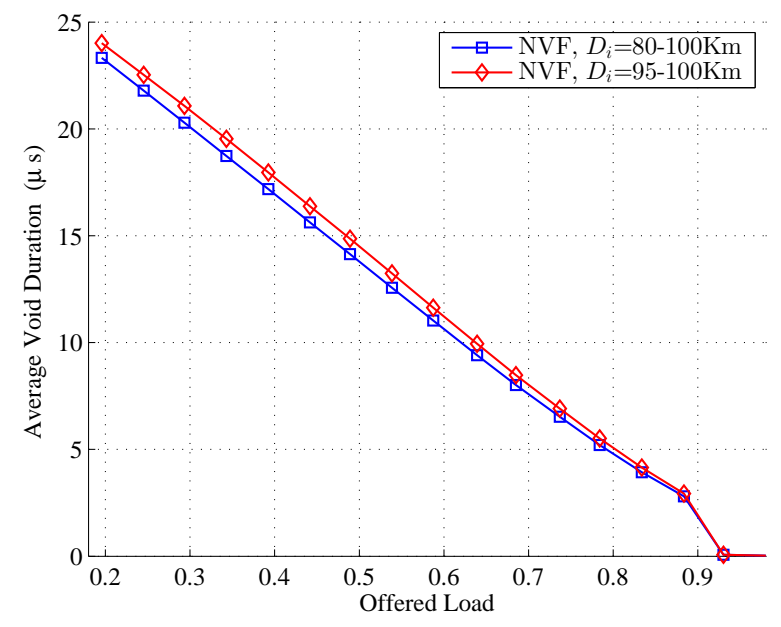

Fig. 6: Void duration between consecutive RBGs.

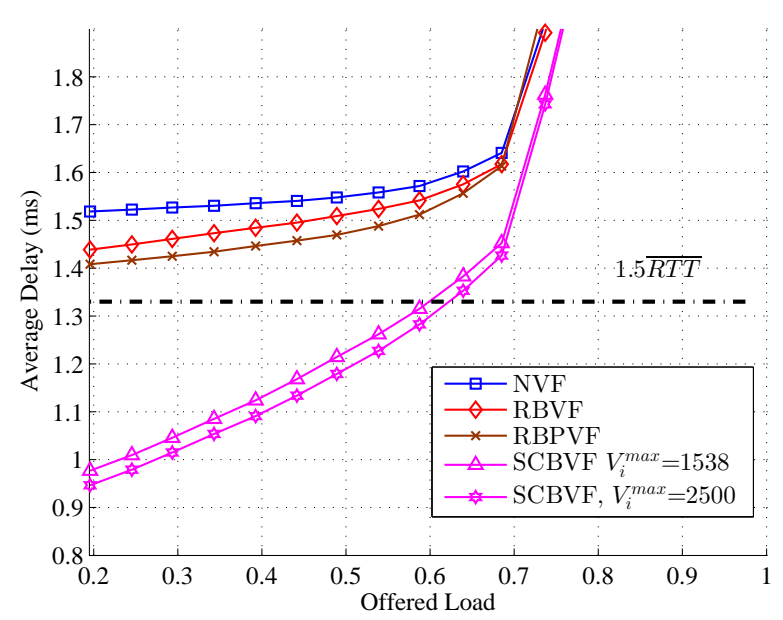

(a)

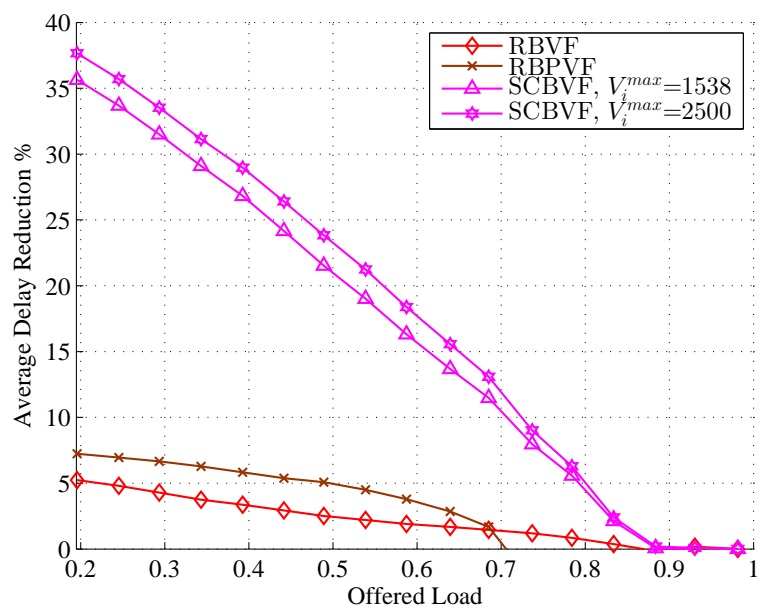

(b)

Fig. 7: (a) Average delay, $D_{i}=80-100 \mathrm{Km}$ (b) Average delay reduction, $D_{i}=80-100 \mathrm{Km}$.

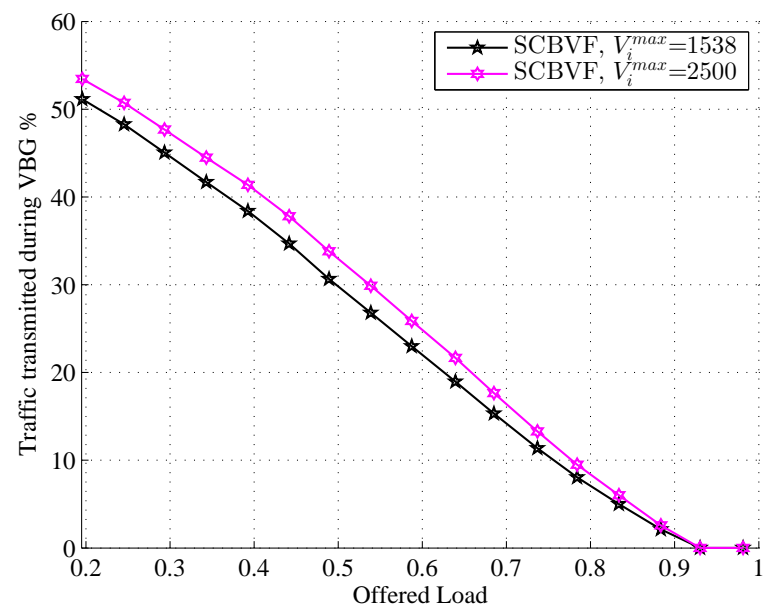

(a)

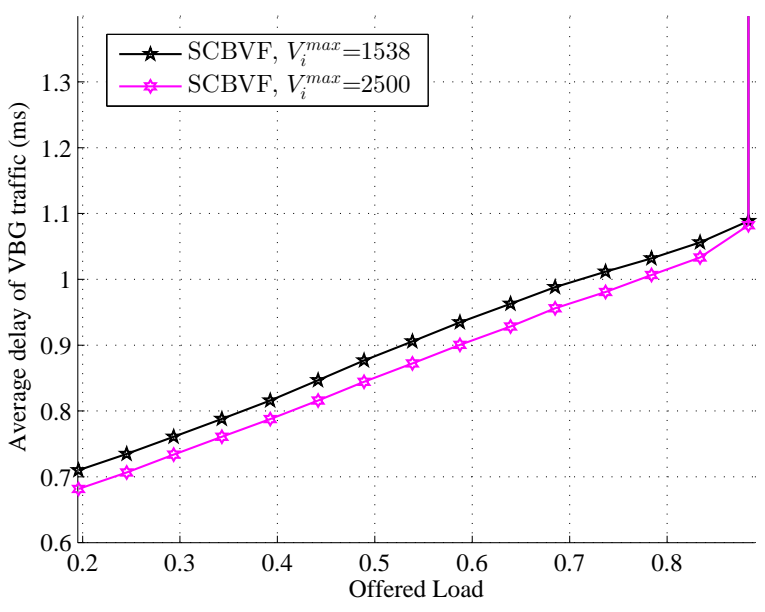

(b)

Fig. 8: For $D_{i}=80-100 \mathrm{Km}$ (a) Traffic $\%$ transmitted during VBG (b)Delay of transmitted packets during VBG.

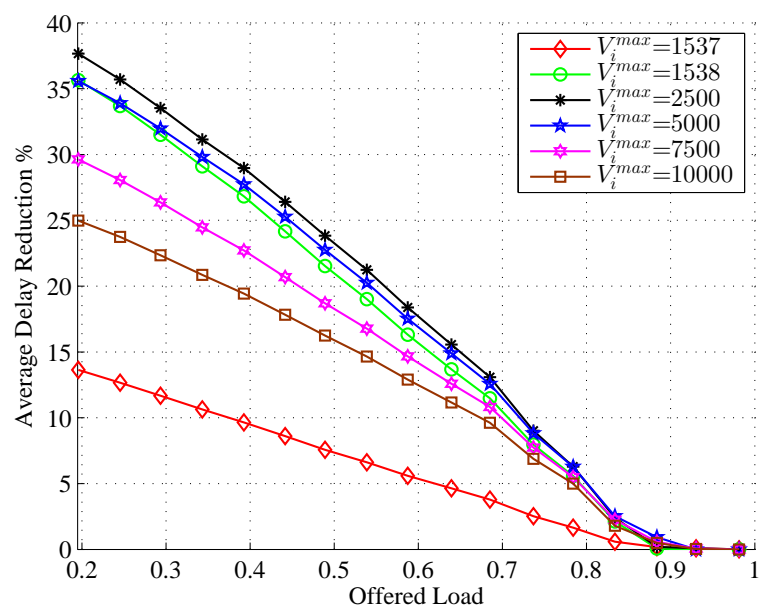

Fig. 9: SCBVF delay reduction ratio $\left(D_{i}=80-100 \mathrm{Km}\right)$. 


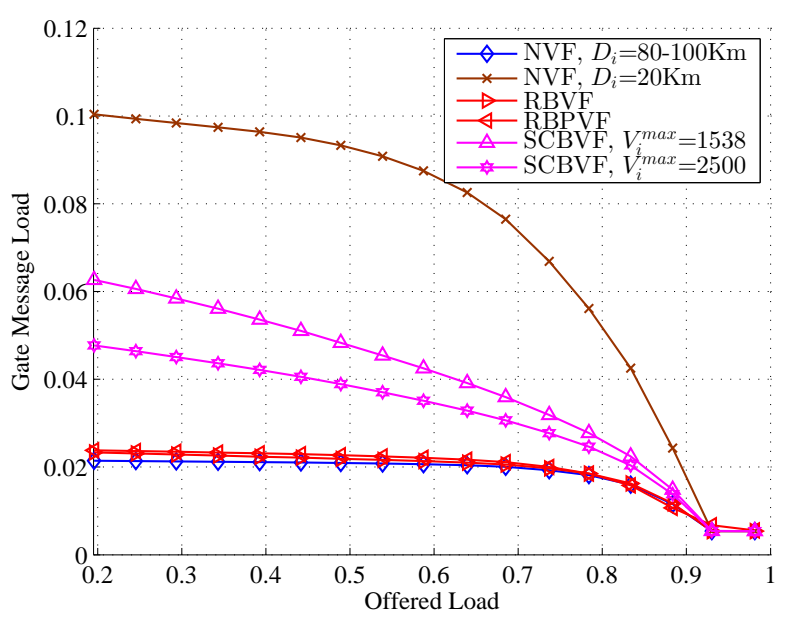

(a)

Fig. 10: Gate message proliferation, $D_{i}=80-100 \mathrm{Km}$.

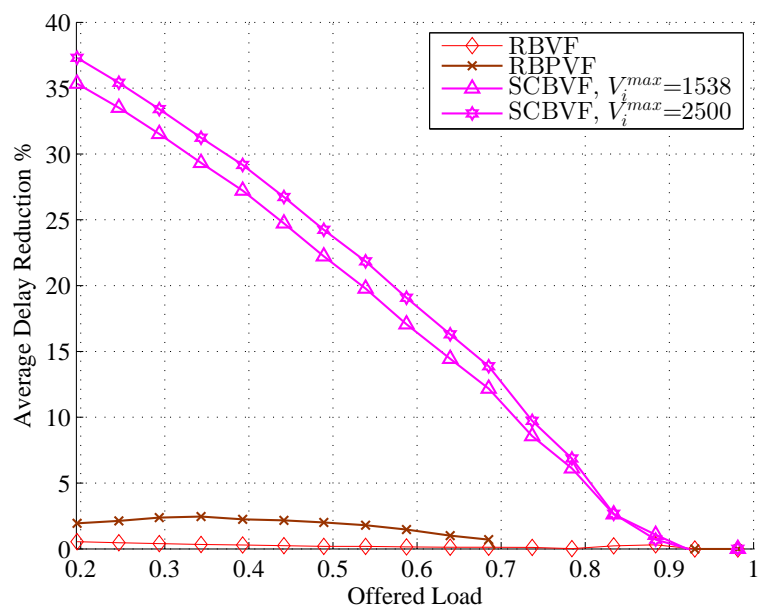

Fig. 11: Delay reduction ratio, $D_{i}=95-100 \mathrm{Km}$.

\section{Performance Evaluation}

\section{A. Simulation Setup}

We consider an LR-EPON with single OLT and 32 ONUs with $10 \mathrm{MB}$ buffer size. The upstream and the downstream transmission rates are symmetric with $1 \mathrm{Gbps}$. Ethernet frames size ranges between 64 to 1518 bytes, (we used the packet size distribution reported in [10], with minimum inter-framegap (IFG) of 12 bytes and preamble of 8 bytes). The incoming traffic is self-similar traffic with long range dependence (LRD) and Hurst parameter 0.8 and packets are served in first-comefirst-served (FCFS) order. $T_{\text {cycle }}^{\max }$ is set to $4 \mathrm{~ms}, t_{g}$ is set to 1 $\mu \mathrm{s}$ and $G_{i}^{\max }=15500$ bytes. The offered load is distributed uniformly over ONUs. We have chosen STP-online-excess as the underlying DBA scheme with excess pool bound of $N G_{i}^{\max }$ bytes. SCBVF is compared against NVF, RBVF, and RBPVF.

\section{B. Numerical Results}

The average void duration of STP-online-excess is shown in Fig.6 for two different distance spans. The results show the void duration decreases with offered load increase. The void duration approximately ranges from 15 to $24 \mu s$ at load below 0.5 . If we consider the total voids per cycle duration $(\approx 1 \mathrm{~ms})$, the idle time duration represents $50-75 \%$ of the cycle length.

Fig.7 shows the delay reduction comparison among NVF, RBVF, RBPVF, and SCBVF for $D_{i}=80-100 \mathrm{Km}$. At 0.7 load and above, all schemes have approximately similar delay. SCBVF is better than NVF, RBVF, and RBPVF for all load range below 0.7 . The delay reduction ratio is calculated based on NVF delay. RBVF achieves delay reduction ratio is up to $5 \%$. RBPVF achieves $7 \%$ delay reduction at low load but it quickly degrades with increased load. SCBVF achieves the largest delay reduction compared to the other schemes. Its delay reduction is up to $35 \%$ and $37 \%$ for $v_{i}^{\max }=1538$ bytes and $v_{i}^{\max }=2500$ bytes respectively. It is also worth mentioning that SCBVF achieves a average of a delay lower than $1.5 \overline{R T T}$ bound.

The reason behind SCBVF excel is shown in Fig.8. It shows two performance measures namely traffic ratio transmitted during VBG and average delay of traffic transmitted during VBG. Fig.8(a) shows that the traffic transmitted during VBGs decreases with the offered load as the available voids becomes shorter. SCBVF achieves up to $52 \%$ for $V_{i}^{\max }=2500$ bytes.

The effect of $V_{i}^{\max }$ on SCBVF is shown in Fig.9. One interesting note to highlight is the significant difference in performance for $V_{i}^{\max }$ equals 1537 and 1538 bytes. This is due to the head of line blocking (HOLB) phenomena. In HOLB, a large frame can block ONU from utilizing all assigned VGBs during one cycle and hence causes performance degradation. Since the largest Ethernet frame requires 1538 bytes to be transmitted including inter-frame-gap and preamble, it is recommended that $V_{i}^{\max }$ should not be less than that value. In Fig.9, SCBVF performance is improving with $V_{i}^{\max }$ increase but this behavior is reversed when $V_{i}^{\max }$ is close to or greater than $G_{i}^{\max }$. One interesting point for future research investigation is how to adapt $K_{B}$ or $V_{i}^{\max }$ with void utilization and VBG traffic ratio to achieve a better performance with daily traffic variations through the access network.

Gate message proliferation is shown in Fig.10. SCBVF has larger gate message load compared to the other schemes, while RBVF is much close to NVF. The gate message load increases when more ONUs are assigned VBGs during voids. This explains why the gate message load decreases with larger $V_{i}^{\max }$. In order to maintain a fair comparison, we compare both SCBVF with NVF at short range $\left(D_{i}=20 \mathrm{Km}\right)$ EPONs. It can be noticed that both SCBVF gate message load is far less than NVF in short range EPONs. Based on this note, we observe that the control message proliferation caused by SCBVF is considered acceptable price for the high average delay reduction ratio they achieve.

Fig.11 shows the average delay reduction compared to NVF 
for $D_{i}=95-100 \mathrm{Km}$. It shows that both RBVF and RBPVF are much affected by the reduced distance span compared to 20 $\mathrm{km}$ distance span presented in Fig.7 (b). However, SCBVF does not face any performance degradation in terms of delay reduction ratio. SCBVF is less sensitive to distance span as it is almost achieves the same reduction ratio. It can be inferred that SCBVF is strong competitor for both RBVF and RBPVF in both cases of long and short differential span distance.

\section{CONCLUSION}

This paper presents a novel void filling approach called Size Controlled Batch Void Filling (SCBVF) for Long-Reach Ethernet passive optical networks (LR-EPONs). SCBVF is an independent polling thread from the running DBA thread(s). Upon detecting a void succeeding a bandwidth grants, SCBVF is invoked to allocate void based grant(s) VBG(s) to fill that void. SCBVF reduces delay by enabling ONUs to early transmit part of their upstream traffic during VBGs. This technique reduces the grant delays and enables bandwidth grants more frequently than both RBVF and RBPVF.

For LRD traffic, numerical results show that SCBVF outperforms NVF, RBVF, and RBPVF and also achieve average delay below $1.5 \overline{R T T}$ bound. SCBVF posses higher gate message load proliferation compared to NVF, RBVF, and RBPVF but it is still less than NVF in short range EPON. A trade off can be done to increase the VBG size and achieve slightly lower delay to reduce the gate load proliferation. Regarding upstream report message proliferation, SCBVF does not add any additional control messages to the underlying DBA scheme. SCBVF performance mainly depends on how much traffic is transmitted during voids, and the average delay of such traffic. Numerical results show that SCBVF has the high VBG traffic ratio. For future research direction, $V_{i}^{\max }$ can be adapted to those parameters to achieve optimum performance with continuous load variations.
SCBVF can be applied without any modification to hybrid time/wavelength division multiplexing PONs (TWDMPONs) for static wavelength allocation. Applying SCBVF for TWDM-PONs with dynamic wavelength allocation must be tied with the wavelength allocation algorithm and is left as a future research direction.

\section{REFERENCES}

[1] G. Kramer, B. Mukherjee, and G. Pesavento, "IPACT: A dynamic protocol for an Ethernet PON (EPON)," IEEE Commun. Mag., vol. 40, no. 2, pp. 74-80, 2002.

[2] A. Mercian, M. McGarry, and M. Reisslein, "Offline and online multithread polling in long-reach PONs: A critical evaluation," J. Lightw. Technol., vol. 31, no. 12, pp. 2018-2028, June 2013.

[3] A. Helmy, H. Fathallah, and H. Mouftah, "Interleaved polling versus multi-thread polling for bandwidth allocation in Long-Reach PONs," IEEE/OSA J. Optical Commun. Netw, vol. 4, no. 3, pp. 210-218, March 2012.

[4] H. Song, B.-W. Kim, and B. Mukherjee, "Multi-thread polling: A dynamic bandwidth distribution scheme in Long-Reach PON," IEEE J. Sel. Areas Commun., vol. 27, no. 2, pp. 134-142, February 2009.

[5] K. Kanonakis and I. Tomkos, "Improving the efficiency of online upstream scheduling and wavelength assignment in hybrid WDM/TDMA EPON networks," IEEE J. Sel. Areas Commun., vol. 28, no. 6, pp. 838848, August 2010.

[6] A. Buttaboni, M. De Andrade, and M. Tornatore, "A multi-threaded dynamic bandwidth and wavelength allocation scheme with void filling for Long-Reach WDM/TDM PONs," J. Lightw. Technol., vol. 31, no. 8, pp. 1149-1157, April 2013.

[7] A. Elrasad and B. Shihada, "Reducing attendance time in LR-EPONs with differentiated services," in INFOCOM 2015 Student Workshop (INFOCOM'15 Student Workshop), Hong Kong, 2015, pp. 70-71.

[8] S. Y. Choi, S. Lee, T.-J. Lee, M. Y. Chung, and H. Choo, "Doublephase polling algorithm based on partitioned ONU subgroups for high utilization in EPONs," IEEE/OSA J. Optical Commun. Netw, vol. 1, no. 5, pp. 484-497, October 2009.

[9] A. Elrasad and B. Shihada, "A practical approach for excess bandwidth distribution for EPONs," in Optical Fiber Communication Conference. OSA, 2014, p. Th1G.6.

[10] D. Sala and A. Gummalla, PON functional requirements: services and performance, in IEEE 802.3ah Meeting in Portland OR, July 2001. [Online]. Available: http://www.ieee802.org/3/efm/public/jul01/ presentations/sala_1_0701.pdf. 